\title{
Diptères nouveaux du Nord de l'Afrique.
}

\section{Par le Dr. J. Villenenve, Rambouillet.}

\section{Helicobosca nigrapex n. sp.}

Caesia ; abdominis segmento ultimo nigro-nitido; alis fuscanis.

De plus grande taille et plus robuste que $H$. muscaria Meig., cette espèce est remarquable par sa coloration d'un noir bleuâtre couverte d'une légère pruinosité cendrée sur le thorax et les 3 premiers segments de l'abdomen; le dernier segment tout entier d'un noir brillant ainsi que le bord postérieur des $2^{\mathrm{e}}$ et $3^{\mathrm{e}}$ segments. Les ailes sont amples, entièrement d'un gris fuligineux plus foncé vers la base et le bord antérieur. La tête d'un gris obscur à reflets blancs est sensiblement plus haute et les yeux plus allongés que chez H. muscaria, mais le péristome est plus étroit et n'a que $1 / 4$ de la hauteur d'oeil. Les antennes un peu plus longues aussi sont d'un noir-âtre; l'ouverture buccale se montre encadrée de rouge.

Une $q$ mesurant $14 \mathrm{~mm}$, prise en mai à Tabarka, dans la collection de Mr. Becker; un autre individu dans la collection de Mr. Bezzi provenant d'Alger, si je me souviens bien.

\section{Sarcotachina umbrinervis n. sp.}

Laete cinerea; thorace trilineato; abdominis segmentis 2 et 3 nigricantibus antice albo-tessellatis, segmento $4^{0}$ albo postice nigro-nitido. Alarum nervis longitud. 1 et 3 ciliatis, nervis transversis fuscedine cinctis. Long. $5-6^{1} / 2 \mathrm{~mm}$.

Tête cendrée, la bande frontale de même couleur chez le ơ teintée d'orangé chez la 우.

Antennes élargies chez la $q$; le $3^{\text {e }}$ article d'un gris roux dans les 2 sexes. Palpes jaunes, épaissis au bout.

Thorax cendré avec une bande noire médiane non interrompue mais s'effaçant en arrière où le thorax présente, en avant du scutellum, une teinte d'un gris terreux chez le $\sigma^{7}$, d'un gris orangé chez la $q$; les bandes latérales noires sont largement interrompues à la suture, en forme de point-virgule. $\mathrm{Dc}=2: 3$; st $=1: 1$.

Scutellum cendré avec 3 soies marginales de chaque côté, le médianes croisées.

Abdomen : $1^{\text {er }}$ segment noir; les $2^{\text {e }}$ et $3^{\text {e }}$ segments d'un noir terne avec le bord antérieur étroitement marqueté de blanc; le $4^{\mathrm{e}}$ segment d'un noir brillant avec une large bande blanche portant en devant 2 petites échancrures noires. Chez la + , cette bande blanche transversale est coupée par une bande dorsale 
orangée. $1^{\text {er }}$ et $2^{\mathrm{e}}$ segments avec 2 soies marginales médianes, $3^{\mathrm{e}}$ et $4^{\mathrm{e}}$ avec une rangée complète.

Appareil génital: Chez le $\sigma^{7}$, callosité du $1^{\text {er }}$ segment d'un noir brillant marqué de rouge sur les côtés; $2^{\mathrm{e}}$ segment également noir mais bordé de blanc en-dessous - chez la $q$, le $1^{\text {er }}$ segment noir avec un point blanc de chaque côté et le $2^{\mathrm{e}}$ segment rouge.

Ailes: Nervures foncées, pâles à la base; les nervures transverses ombrées, la nerv. transverse postérieure plus rapprochée de la petite transverse. $1^{\text {ère }}$ nervure ciliée sur toute sa longueur, la $3^{\text {ème }}$ ciliée au delà de la petite nervure transverse. Epine costale longue.

Griffes et pelotes du $\sigma^{7}$ très allongées.

Un $\sigma^{\top}$ et une + , pris en mai à Tunis, dans la collection de Mr. B e cke r.

\section{Sarcotachina aegyptiaca n. sp.}

Aspect d'une Hilarella.

Tête blanche avec le front d'un gris cendré dans les deux sexes. Palpes jaunes.

Les bandes noires du thorax sont presque entièrement effacées, mais on y voit une tache noire médiane un peu allongée et très nette, située en arrière de la suture. $\mathrm{Dc}=2: 3$ dont le pore d'insertion est fortement auréolé de noir; st $=1: 1$.

Scutellum marqué de noir aux angles antérieurs; 3 soies marginales de chaque côté, les médianes croisées.

Abdomen: $1^{\text {er }}$ segment avec une seule soie latérale; il a la même coloration que les suivants mais sans aucune tache. $2^{\mathrm{e}}$ et $3^{\text {e }}$ segments avec 2 soies marginales médianes insérées chacune sur un gros point noir, et $1-2$ soies latérales sur un point noir plus petit; au bord antérieur de ces 2 segments existent encore 2 points noirs très éloignés et ne portant pas de soie. $4^{\mathrm{e}}$ segment avec 4 taches noires marginales séparées, munies d'une forte soie. En outre, l'abdomen a 3 grosses taches triangulaires sur les flancs.

Appareil génital: Chez le $\sigma^{x}, 1^{\text {er }}$ segment d'un noir brillant; $2^{\mathrm{e}}$ segment rouge - chez la + , le $1^{\mathrm{er}}$ segment cendré avec une fine ligne noire dorsale, le reste rouge.

Ailes: $1^{\text {ère }}$ nervure nue; $3^{\mathrm{e}}$ nerv. ciliée jusqu'à la petite nervure transverse; nerv. transverse postérieure à égale distance des deux autres; épine costale longue.

Pattes d'un gris obscur: griffes et pelotes très allongées chez le $\sigma^{7}$. Longueur : $7-8 \mathrm{~mm}$.

D'Egypte (Assuan, Siala, Alexandrie etc.), de février à mai, dans la collection de Mr. B e cke r et la mienne. J'ai vu aussi un individu dans la collection de $\mathrm{Mr}$. B e z zi. 
IV. Wohlfahrtiodes nuda n. gen. n. sp.

Cette espèce a tout à fait l'aspect de Wohlfahrtia, mais je crois devoir la ranger dans un genre distinct pour les raisons suivantes: l'abdomen a un dessin différent, il est entièrement nu à l'exception du dernier segment qui possède une rangée marginale complète de soies fines; enfin, l'armature génitale du $\sigma^{7}$ s'éloigne par sa conformation, à savoir: le forceps épais et court, terminé, au lieu de branches, par 2 denticules visibles seulement avec une bonne loupe et sur lesquelles viennent s'appuyer les sommets des paralobes (parameren). Ceux-ci sont développés et ont une forme triangulaire. Le pénis court a son extrêmité discoïde.

ơ. Gris-blanchâtre; tête blanche, bande frontale obscure et à reflet gris; pas de soies orbitaires-externes; antennes noires, chète de même couleur, nu, épaissi dans les $2 / 5$ puis brusquement aminci; gênes nues; palpes noirâtres. Thorax rayé de noir comme chez Wohlfahrtia; $\mathrm{d} c=2: 3$, les premières faibles; st $=1: 1$.

Abdomen : $1^{\text {er }}$ segment un peu obscur, $2^{\mathrm{e}}$ et $3^{\mathrm{e}}$ segments ayant chacun une seule tache noire, médiane, en triangle allongé, ces taches formant une bande anguleuse très nette qui s'évanouit sur le dernier segment. Ventre d'un brun cuivreux. Hypopygium à segments cendrés; le forceps obscur, les paralobes et le pénis rouges.

Ailes comme chez Wohlfahrtia.

Pattes avec les griffes de devant peu allongées. Taille: $9 \mathrm{~mm}$.

De Assuan (Egypte) où Mr. B e ck e r l'a prise en février sur des chameaux; j'ai reçu de lui un exemplaire pour ma collection.

\section{Berichtigung.}

In dem Referat über Dr. Arnold Pagenstecher, Die geographische Verbreitung der Schmetterlinge, Heft I, Seite 123 u. 124, sind leider einige Druckfehler stehen geblieben, da ich wegen der unmittelbar bevorstehenden Abschliefsung des Heftes keine Korrektur mehr lesen konnte. Seite 124,17 . Zeile von oben ist zu lesen "Syntomididen" und "Lithosiiden" für "Syntaniden" und "Lithoriden", 14. Zeile von unten (im Referat) "Hestia" für "Hastia“.

K. G r ün be r $g$. 


\section{$2 \mathrm{BHL}$ Biodiversity Heritage Library}

Villeneuve, Joseph. 1910. "Diptères nouveaux du Nord de l'Afrique." Deutsche entomologische Zeitschrift 1910(2), 150-152.

https://doi.org/10.1002/mmnd.4801910204.

View This Item Online: https://www.biodiversitylibrary.org/item/110145

DOI: https://doi.org/10.1002/mmnd.4801910204

Permalink: https://www.biodiversitylibrary.org/partpdf/236006

\section{Holding Institution}

California Academy of Sciences

\section{Sponsored by}

California Academy of Sciences Library

\section{Copyright \& Reuse}

Copyright Status: Public domain. The BHL considers that this work is no longer under copyright protection.

This document was created from content at the Biodiversity Heritage Library, the world's largest open access digital library for biodiversity literature and archives. Visit BHL at https://www.biodiversitylibrary.org. 